\title{
Scar pregnancy and spontaneous rupture uterus - a case report
}

\author{
Ahmed Mosad, Ahmed Altraigey \\ Department of Obstetrics and Gynaecology, Benha University, Benha, Egypt
}

Our objective is to spotlight the management of first trimester rupture uterus that is life-threatening as it is very rare and difficult to be included in the differential diagnosis of acute abdomen in early pregnancy. We report a case of a pregnant woman in her first trimester who presented with sudden onset of abdominal pain, severe vaginal bleeding and repeated syncopal attacks.

A 19 year-old woman G2 P1 + 0 presented at 11 weeks of gestation to emergency unit of Benha University hospitals complaining of sudden onset generalized abdominal pain, severe vaginal bleeding and repeated syncopal attacks. By history, she was delivered through CS six months before her presentation to our hospital. Also, intrauterine contraceptive device (IUCD) was applied five months later and expelled spontaneously a week later. The case has no relevant medical history, by general examination; she was conscious with extremely pale look. Her vital signs showed blood pressure of $80 / 50 \mathrm{~mm} \mathrm{Hg}$, pulse of $120 \mathrm{bpm}$ and $37.2^{\circ} \mathrm{C}$ temperature. Her respiratory rate was 27 per minute. On abdominal examination there were generalized tenderness and rebound tenderness all over the abdomen with shifting dullness on percussion. Urine pregnancy test was positive and serum beta human chorionic Gonadotropin (b-HCG) sample was sent to laboratory.

Her laboratory investigations were satisfactory. Pelvi-abdominal ultrasonography showed empty uterus, single viable foetus about 11 weeks within a protruding gestational sac, marked free intra-abdominal fluid collection. Clear informed consent was obtained from the couple after full counselling about the clinical condition and the management plan.

Emergency laparotomy through pfannensteil incision was carried out after stabilizing the patient clinical condition and cross-matching four units of packed red blood cells ( $p-R B C s$ ). The operative findings corresponded well with ultrasound findings. There was intact single gestational sac protruding through ruptured scar of lower uterine segment (LUS) (Fig. 1). The foetus within it was showing signs of viability by movements (Fig. 2).

The gestational sac was removed and sent for histo-pathological examination as well as most of the trophoblastic tissues adherent and invading the wall of LUS (Fig. 3). The uterine defect was repaired into three layers using $2 \backslash 0$ Vicryl.

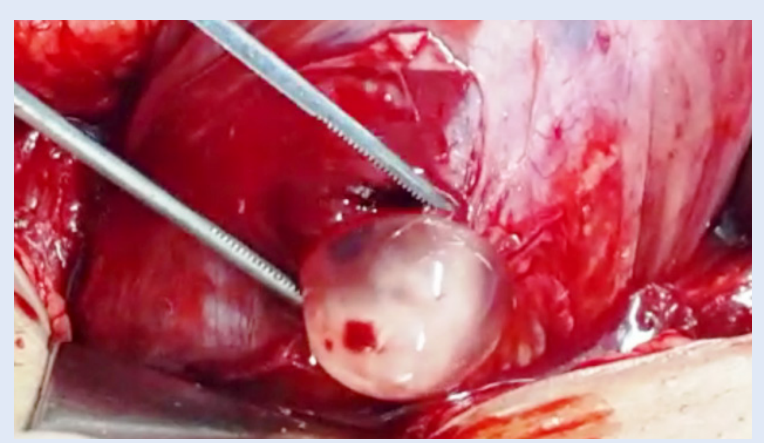

Figure 1. Figure shows the intact gestational sac protruding through a defect in the scarred lower uterine segment

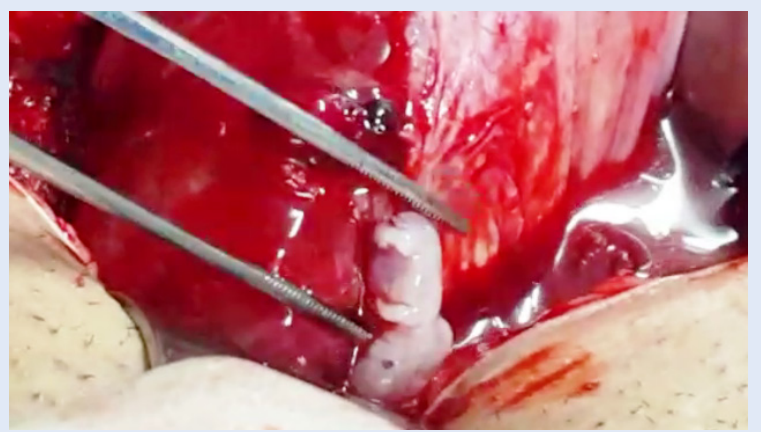

Figure 2. Figure shows a fetus at 11 weeks of gestation after disturbing the scar pregnancy 
Bilateral uterine artery ligation was done to secure haemostasis properly. Peritoneal washing was done and mass closure of the abdomen after inserting two pelvic drains. The case received three units of $\mathrm{p}$-RBCs intraoperatively.

The patient was admitted to intensive care unit for two days with a close monitoring. Her initial b-HCG was $6982 \mathrm{mIU} / \mathrm{mL}$. She was discharged home in a good condition five days after operation. She was followed up through weekly serial b-HCG readings that reached non-pregnant level on third week and remained so for nine months now. The case used combined oral contraceptive pills regularly postoperatively.

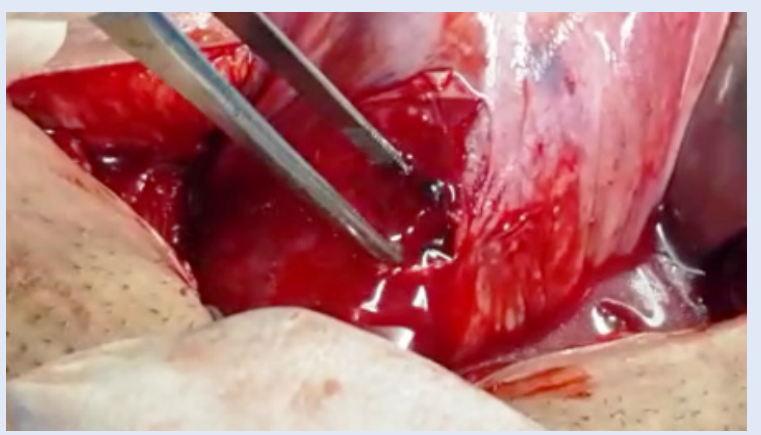

Figure 3. Figure shows the site of uterine rupture due to invading trophoblastic tissues of the scar pregnancy 